> Gastric carcinoma (GC) with gastrointestinal stromal tumor (GIST) is encountered very rarely in the clinic, and few cases have been reported in the literature. Here, we present a case involving a 72-year-old man who was diagnosed with gastric antrum adenocarcinoma accompanied by neuroendocrine differentiation and a GIST in the fundus, according to a preoperative examination and postoperative pathology. The patient then underwent a distal radical gastrectomy and GIST resection. After the operation, the patient was administered combined chemo-radiotherapy and subsequently underwent a 9-month followup examination. The gene mutations involved in this case were explored via high-throughput sequencing. The high-throughput gene mutation analysis indicated an exon5 mutation in the TP53 gene and copy number amplification of FGF19, CCND1, and FGFR2 in the gastric antrum adenocarcinoma. A gene sequencing analysis of the gastric fundus stromal tumor demonstrated an exonll non-frame shift deletion mutation in the KIT gene. These findings suggested that this patient's cancer might be sensitive to AZD1775 (a TP53-targeted drug) or targeted drugs such as FGF19, CCND1 and FGFR2, and should be sensitive to imatinib. <

Key words: Gastric carcinoma (GC); Gastrointestinal stromal tumor (GIST); Gene mutation; High-throughput sequencing.

Gastric carcinoma (GC) is a common gastric malignant tumor originating from epithelial tissues, and gastrointestinal stromal tumor (GIST) is a type of gastrointestinal tumor with malignant potential [1]. GIST originates from the interstitial cells of Cajal (ICCs; also known as gastrointestinal pacemaker cells[2]) or mesodermal mesenchymal stem cells that are more primitive and have multiline age differentiation

\section{Gastric \\ carcinoma with a gastrointestinal stromal tumor}

\section{A case report and literature review}

Sen Liu ${ }^{1}$, Hongyu Liu ${ }^{2}$, Yunlong Dong ${ }^{2}$, Fengbiao Wang ${ }^{1}$, Huijuan Wang ${ }^{1}$, Jun Chen ${ }^{2,3^{*}}$

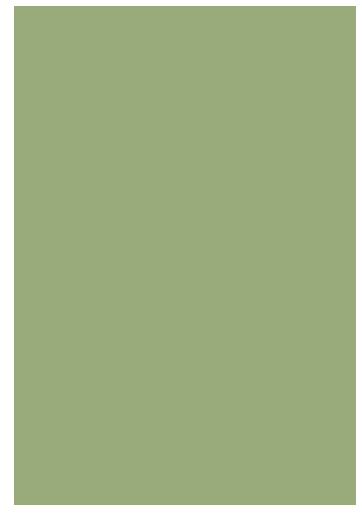

${ }^{1}$ Department of Gastrointestinal Surgery, Tianjin 4th Central Hospital, Hebei District, Tianjin 300140, China.

${ }^{2}$ Tianjin Key Laboratory of Lung Cancer Metastasis and Tumor Microenvironment, Tianjin Lung Cancer Institute. ${ }^{3}$ Department of Lung Cancer Surgery, Tianjin Medical University General Hospital, Heping District, Tianjin 300052, China.

potential. Cases involving GC with huntercj2004@qq.com GIST are rare in clinical practice, and there are only a few case reports in medical literature.

\section{Case presentation}

A 72-year-old male patient was admitted with the chief complaint of upper abdominal pain for 1 month; the pain predominantly occurred at night or with hunger and was accompanied by a sour regurgitation and weight loss. He had no family history of malignant tumors. Routine blood, routine urine, and blood biochemical tests were normal, and the tumor marker test results were all within normal ranges, including alpha-fetoprotein (AFP), serum ferritin (SF), carcinoembryonic antigen (CEA), carbohydrate antigen 19-9 (CA 19-9), carbohydrate antigen 72-4 (CA 72-4), prostate-specific antigen (PSA), neuron-specific enolase (NSE), cytokeratin-19 fragment (CYFRA 19), and carbohydrate antigen 15-3 (CA 15-3). Upper abdominal computed tomography (CT) plain and enhanced scans revealed ulcerated lesions in the gastric antrum with apparent enhancement that were consistent with GC (Figure IA), as well as clearly bordered and enhanced exogenous nodes in the gastric fundus that indicated a probable stromal tumor (Figure 1B). Gastroscopy revealed a giant, yellow-coated, and necrotic ulcer at the bottom of the gastric antrum, and a gastroscopic histopathological biopsy indicated adenocarcinoma; there was normal mucosa in the gastric fundus (Figure IC). 

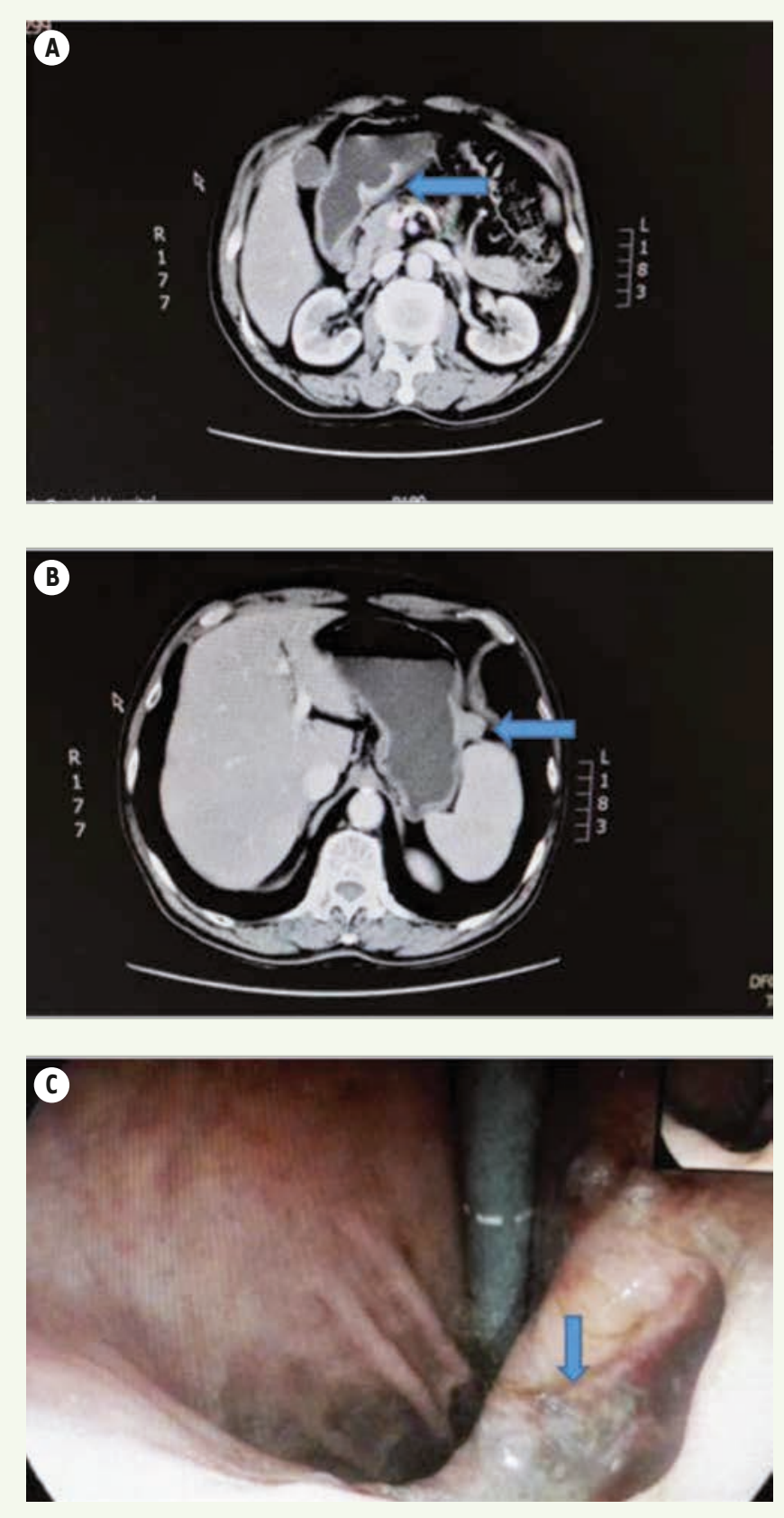

Figure 1. CT and gastroscopy indicate GC with GIST. (A) CT indicates an ulcerated $\mathrm{GC}$ lesion in the gastric antrum; (B) CT indicates an exogenous stromal tumor in the gastric fundus; (C) Gastroscopy revealed a large ulcer in the gastric antrum.

During surgery, in the lesser curvature near the gastric antrum, a hard tumor measuring $4 \times 4 \mathrm{~cm}^{2}$ with shrunken serosa was revealed that indicated GC, accompanied by enlarged adjacent lymph nodes; thus, a distal radical gastrectomy was performed. A $2-\mathrm{cm}$ exogenous soft lump was found near the greater gastric curvature, and the adjacent gastric wall was normal, without any lymph node enlargement; these findings were indicative of GIST, so a GIST resection was performed. The histopathology of the gastric antrum showed moderately differentiated adenocarcinoma with neuroendocrine differentiation that invaded into the deep muscle layer, and the ratio of the omental lymph node metastasis was $1 / 10$. Immunohistochemistry demonstrated the following: CK (+), CK8/18 (+), and CgA (-); SyN: focus $(+)$ and CD56 (-); and CD34: lymphovascular space (+) (Figure 2). The histopathology of the gastric fundus indicated GIST; features included spindleshaped cells, no significant atypia, no obvious signs of necrosis, mitotic 1/50HPF. Immunohistochemistry demonstrated the following: CDl17 (+), WTl (-), Ki67 $(-), \mathrm{BCl}-2(-)$, CD34 (+), SMA focus $(+), \mathrm{S}-100$ sparse $(+)$, and vimentin $(+)$. All margins were negative. Combining these immunohistochemical results with the preoperative examination, intraoperative findings, and postoperative pathology, a diagnosis of gastric antrum adenocarcinoma with neuroendocrine differentiation accompanied by a gastric fundus stromal tumor was made and the patient's pathological stage was Stage Ila $\left(\mathrm{T}_{2} \mathrm{~N}_{1} \mathrm{M}_{0}\right)$.

The patient recovered well and was discharged one week after his operation. Four weeks postoperatively, chemotherapy was started with a FOLFOX4 regimen (oxaliplatin, $85 \mathrm{mg} / \mathrm{m}^{2}$ IVGTT, day $\mathrm{l}$; leucovorin [CF], $200 \mathrm{mg} / \mathrm{m}^{2} / \mathrm{d}$ IVGTT, 2 hrs before administration of 5 -FU,

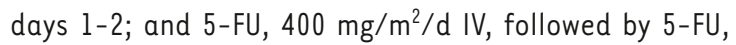
$600 \mathrm{mg} / \mathrm{m}^{2} / \mathrm{d}$ IVGTT, for $22 \mathrm{~h}$, days $\mathrm{l}-2$ ). The treatment was repeated after 14 days, and there were a total of 12 cycles. Considering GIST, oral imatinib was administrated at the same time. The patient insisted on chemotherapy and targeted treatment, and he regularly underwent reexamination for tumor markers and obtained follow-up gastroscopic and abdominal CT examinations. There were no recurrences or distal metastases detected after eighteen months of follow up.

To further investigate the molecular abnormalities of the tumor, we detected tumor-related gene mutations in the GC and GIST lesions using next-generation sequencing (NGS) like our previous work [3] (Guangzhou Burning Rock Medical Examination Institute Co, Ltd, China). The driver genes included: ALK, ARAF, AKTI, APC, ATM, $B I M, B R C A 1, B R C A 2, B R A F, C C N D 1, C D K 4, C D K 6, C D K N 2 A$, CTNNB1, CYP2D6, DDR2, DPYD, ERBB3, ERBB4, EGFR, ERBB2, FGF3, FGF4, FGF19, FGFR1, FGFR2, FGFR3, FLT3, HRAS, JAK1, JAK2, KDR, KIT, MYC, MAP2K1, MET, MTOR, NTRK1, NTRK2, NTRK3, NRAS, KRAS, PDGFRA, PIK3CA, PTCH1, PIK3CA, RAF1, RET, ROS1, STK11, TP53, TSC1, TSC2, and UGTIAl. No mutations in the KRAS, HRAS, NRAS, BRAF, EGFR, ERBB2, KIT, PDGFRA, MET, or APC gene were detected in this patient's $G C$ lesion. No mutations in the $\varepsilon G F R, A L K, E R B B 2, B R A F, M E T, R E T, R O S 1$, or KRAS gene were detected in the GIST lesion. But there were 


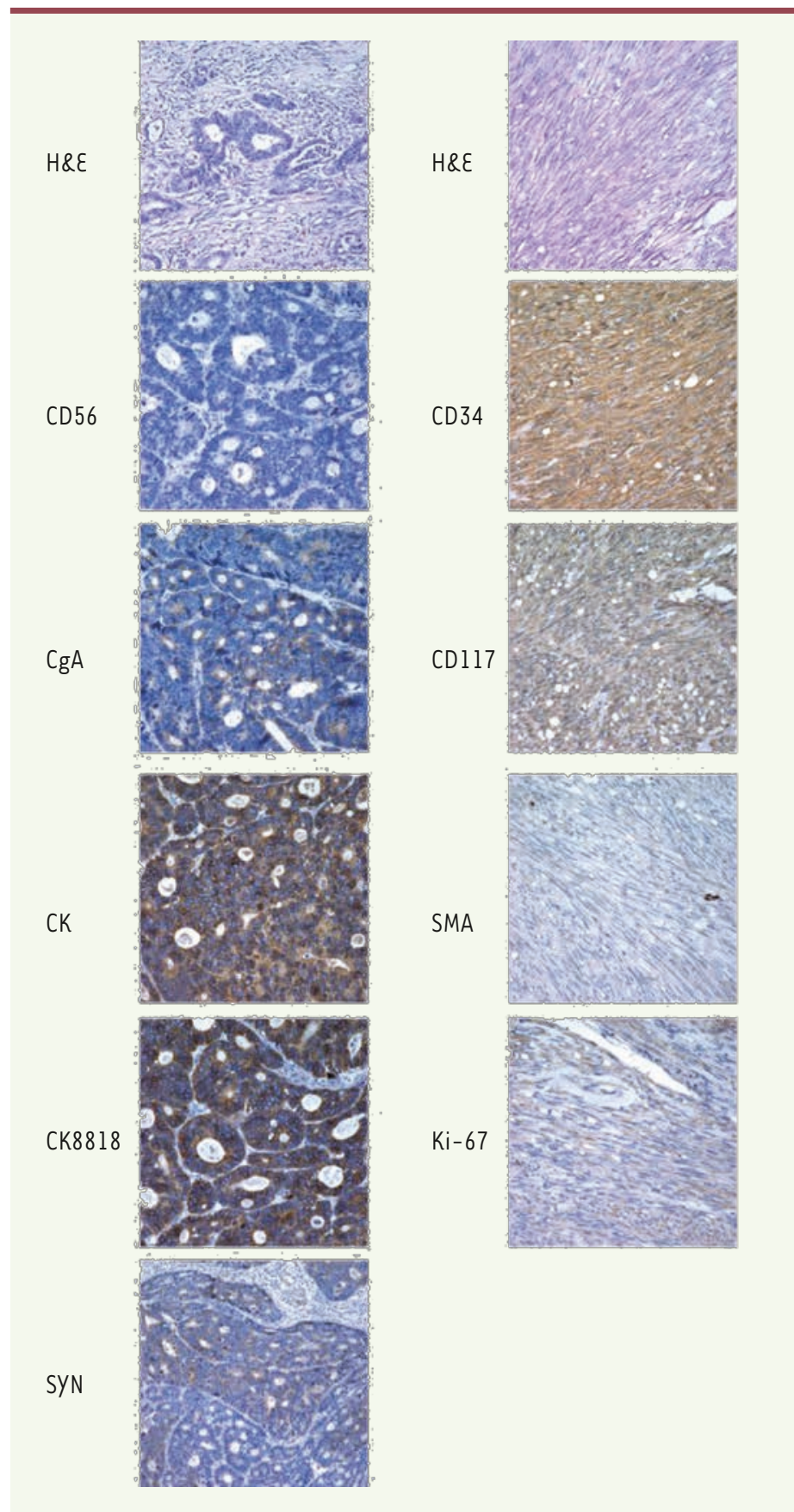

Figure 2. Histopathology. $\mathrm{H} \varepsilon$ staining indicates gastric adenocarcinoma with neuroendocrine differentiation and GIST. The expression of CD56, CgA, CK, CK8818, and SYN was detected in the gastric adenocarcinoma tissues with neuroendocrine differentiation, and CD34, CD117, SMA, and Ki-67were detected in the GIST tissues with immunohistochemical staining. (Magnification of $400 x$ ).

mutations/amplification of the TP53, FG19, CCND1, and FGFR2 genes in the GC lesion, as well as an exon 11 non-frame shift deletion mutation, Cl652-1666 del, in the KIT gene in the GIST lesion, as shown in Tablel. Therefore, GC and GIST have markedly different gene mutations and a different pathogenesis at a molecular level.

\begin{tabular}{lll}
\hline Position & Gene & Mutation/Abnormality \\
\hline GC & & \\
& TP53 & $\begin{array}{l}\text { Exon } 5 \text { nonsense mutation, } \\
\text { c538G>T }\end{array}$ \\
& FGF19 & Copy number amplification \\
& CCND1 & Copy number amplification \\
\hline & FGFR2 & Copy number amplification \\
\hline GIST & & \\
\hline & KIT & $\begin{array}{l}\text { Exon } 11 \text { non-frame shift deletion } \\
\text { mutation, Cl652-1666 del }\end{array}$ \\
\hline
\end{tabular}

Table 1. Gene mutations/abnormalities in GC and GIST.

\section{Discussion}

$\mathrm{GC}$ is a common gastric malignant tumor originating from epithelial tissues and demonstrates regional differences in China. Its age of onset is $\geq 50$ years, and the ratio of males/females with GC is $2: 1$. Precancerous lesions of $\mathrm{GC}$ include gastric polyps, and chronic atrophic gastritis, as well as gastric remnants from a subtotal gastrectomy. Diagnosis of GC depends on pathology and immunohistochemical analyses. GIST is a mesenchyme-derived tumor that predominantly involves spindle cells; lacks definite muscularity or neural differentiation; is difficult to determine the direction of differentiation; and frequently occurs in the gastrointestinal tract, mesentery, and peritoneum [4]. Immunological manifestations of GIST typically involve CD117 (80\%-100\%; +), CD34 (56\%-83\%; +), and $c$-kit gene mutations (+) [5], which indicates that high expression of CD117 and CD34 might be helpful for GIST screening and diagnosis. Referring to the classification criteria of Emory et al. [6], GIST is classified as malignant, potentially malignant, or benign. Malignant classification requires that the tumor has: 1) distant metastasis and 2) invasion. Classification as potentially malignant requires that the tumor cells: 1) are tightly arranged and actively growing and 2) have obvious abnormalities; 3 ) the karyokinetic cells are $\geq 5 / 50$ high power fields (HPF), and the tumor is 4) necrotic and 5) has a diameter of $>5.5 \mathrm{~cm}$. Malignant GIST has two malignant indicators or more than two potentially malignant indicators and one malignant indicator; potentially malignant GIST has only one potentially malignant indicator; and benign GIST has none of the abovementioned indicators. In this case, the gastric fundus stromal tumor was benign. 


\begin{tabular}{|c|c|c|c|c|c|}
\hline Authors and Year & $\mathbf{N}$ & $\begin{array}{l}\text { Sex/Age } \\
\text { (year) }\end{array}$ & $\begin{array}{l}\text { GC } \\
\text { Position/Diameter } \\
\text { (cm)/TNM Stage }\end{array}$ & $\begin{array}{l}\text { GIST } \\
\text { Position/Diameter } \\
\text { (cm)/Risk Level }\end{array}$ & Follow-up (month) \\
\hline \multirow[t]{5}{*}{ Maiorana A, et al, 2000 [7] } & 1 & $F, 81$ & $\mathrm{CA} / 4.0 / \mathrm{T} 2 \mathrm{bNOMO}$ & $\mathrm{F} / 5.0$ /potentially malignant & 21 , no tumor- related death \\
\hline & 2 & $F, 79$ & $\mathrm{~A} / 2.0 / \mathrm{Tl} \mathrm{bNOMO}$ & $\mathrm{P} / 6.0 /$ malignant & 54, tumor-free survival \\
\hline & 3 & $M, 75$ & $\mathrm{~A} / 4.0 / \mathrm{T} 2 \mathrm{bN} 1 \mathrm{MO}$ & A/5.0/potentially malignant & 12 , tumor-free survival \\
\hline & 4 & $\mathrm{~F}, 79$ & $\mathrm{P} / 1.2 / \mathrm{T} 2 \mathrm{aN} 1 \mathrm{MO}$ & C/5.0/potentially malignant & 28, tumor-free survival \\
\hline & 5 & $M, 79$ & $\mathrm{~A} / 2.0 / \mathrm{T} 2 \mathrm{aNOMO}$ & c/0.6/benign & 75, tumor-free survival \\
\hline Liu SW, et al, 2002 [8] & 1 & $M, 70$ & $\mathrm{CA} / 8 / \mathrm{T} 3 \mathrm{~N} 1 \mathrm{Ml}$ & C/NM/NM & $\begin{array}{l}3 \text {, death because of postope- } \\
\text { rative complications }\end{array}$ \\
\hline \multirow[t]{2}{*}{ Bircan S, et al, 2004 [9] } & 1 & $M, 71$ & $\mathrm{~A} / 5.7 / \mathrm{T} 3 \mathrm{~N} \times \mathrm{MO}$ & C/0.5/very low & NM \\
\hline & 2 & $M, 77$ & $\mathrm{C} / 7.5 / \mathrm{T} 2 \mathrm{~N} 1 \mathrm{MO}$ & CA/0.6/very low & NM \\
\hline Lin YL, et al, 2006 [10] & 1 & $F, 70$ & $\mathrm{GA} / 1.7 / \mathrm{TINxMO}$ & F/l.1/NM & 14 , tumor-free survival \\
\hline \multirow[t]{2}{*}{ Wronski M, et al, 2006 [11] } & 1 & $F, 64$ & A/5/T4NOMO & C/2/low & NM \\
\hline & 2 & $M, 66$ & GA/I/TINOMO & $\mathrm{C} / 1 /$ very low & NM \\
\hline $\begin{array}{l}\text { Katsoulis IE, et al, } 2007 \\
\text { [12] }\end{array}$ & 1 & $F, 88$ & $\mathrm{C} / 10 / \mathrm{T} 3 \mathrm{~N} 2 \mathrm{MO}$ & A/0.9/benign & NM \\
\hline
\end{tabular}

Table 2. Summary of the pathological characteristics in the case reports on GC with GIST. Note: C, corpus; CA, cardia; F, fundus; $A$, antrum; $P$, pylorus; $\mathrm{GA}$, gastric angle; NM, not mentioned.

GC with GIST is rare, and its morbidity is only $0.53 \%$ [7]. There are only a few case reports on GC with GIST worldwide [8-13], perhaps because GIST is small and often masked by the clinical manifestations of GC (Table 2). The etiology of GC with GIST is unknown, but there are several hypotheses. From a clinical perspective, the occurrence of GC with GIST is random, but some studies have shown that it could be associated with certain common carcinogenic factors $[14,15]$. Another studies believed that a gene mutation might lead to an interaction between two types of adjacent tissues, along with a disturbance in the regulation of mesothelial and epithelial cell growth, thus inducing different tumors in two types of tissues in the same organ $[8,10,16]$. Conventional treatment for primary GC includes early surgery plus neoadjuvant chemotherapy or various combinations of adjuvant chemotherapy and radiotherapy after surgery. In addition to surgery, postoperative targeted drug treatment is required for GIST because of its insensitivity to conventional chemotherapy and radiotherapy [17]. The NGS results showed that the patient had a nonsense mutation in the TP53 gene and a copy number amplification of the FGF19, CCNDI, and FGFR2 genes in the GC lesion, and no significant abnormalities in any other genes were detected, indicating poor prognosis. In the GIST lesion, there was only an exon 11 non-frame shift deletion mutation in the KIT gene and PDGFRA (-). We could see a marked difference between GC and GIST that further suggests that these two diseases can present simultaneously in the same patient but have different origins of pathogenesis at a molecular level. It provides a basis for exploring precisely targeted therapeutic drugs. The exon 5 nonsense mutation in the TP53 gene in the GC lesion indicated that GC might be sensitive to AZD1775 (a TP53-targeted drug). The frequency of occurrence of an FGFR2 gene amplification is $4-6 \%$ in GC patients. FGFR2 inhibitors (e.g., ponatinib and AZD4547) have an inhibiting effect on FGFR2-amplified cell lines in vitro, but its superiority to chemotherapy alone has not been confirmed in clinical trials. The overexpression of both CCNDI and FGF19 indicates that patients have poor prognosis. Cetuximab, a recognized targeted drug, might not work because the GC lesion was negative for EGFR in this case, indicating that the use of targeted drugs should be based on treatment individualization. In this case, the GIST lesion had an exon 11 non-frame shift deletion mutation in the KIT gene; thus, imatinib, a KIT inhibitor, might be effective. We reviewed the published medical literature on GC with GIST. Some investigators propose that treatment of these patients should focus on the GC, and that efficacy can be predicted by evaluating the GC prognosis [18]. For patients with concurrent small GIST that has been resected, there is no need for oral imatinib, but rather follow-up treatment for gastric cancer [19]. Some investigators believe that the patients with GIST should be considered a poor prognosis when complicated with $G C$, regardless of risk level; therefore, the survival time of these patients primarily depends on the long-term efficacy of treatment for GC [20]. Since prognosis depends mainly on the GC, for which surgery is the first treatment of choice, appropriate 
chemotherapeutic regimens can be selected according to the pathological results of the GC. Furthermore, suitable targeted molecular treatment may be used based on a molecular analysis.

\section{Conclusions}

GC with GIST is rare, and its morbidity is very low. The molecular analysis of this case indicates that there are different mutations between GC and GIST. The survival time of this disease mainly depends on GC characteristics; and the primary and most efficient treatment is surgery. $\diamond$

\section{ETHICS APPROVAL AND CONSENT TO PARTICIPATE}

The patient granted written informed consent for publication of this manuscript and the accompanying images, and the study protocol was approved by the clinical research ethical review board of the Tianjin Medical University General Hospital.

\section{CONSENT FOR PUBLICATION}

Not applicable

\section{AVAILABILITY OF DATA AND MATERIAL \\ Not applicable}

\section{COMPETING INTERESTS}

The authors declare no competing interests.

\section{FUNDING}

This study was supported by grants from the National Natural Science Foundation of China (81372306), the Tianjin Natural Science Foundation (16JCZDJC34200, 13JCYBJC22600), and the municipal key laboratory construction project of Tianjin innovative platform special (16PTSYJC00160). Funding sources had no role in study design, data collection, or analysis, nor in the decision to publish or in the preparation of the manuscript.

\section{AUTHORS CONTRIBUTIONS}

$S L, H Y L$ and $Y L D$ collected all data and wrote the manuscript. SL, FBW and HJW performed the surgical procedure and treated the patient. JC contributed to data analysis and revised the manuscript. All authors have read and approved the final manuscript.

\section{ACKNOWLEDGEMENTS}

Not applicable

\section{REFERENCES}

1. Dow N, Giblen G, Sobin LH, Miettinen M: Gastrointestinal stromal tumors: differential diagnosis. Seminars in diagnostic pathology 2006, 23(2):111-119.

2. Kindblom LG, Remotti HE, Aldenborg F, Meis-Kindblom JM: Gastrointestinal pacemaker cell tumor (GIPACT): gastrointestinal stromal tumors show phenotypic characteristics of the interstitial cells of Cajal. The American journal of pathology 1998, 152(5):1259-1269.

3. Dong M, Liu J, Song Z, Li X, Shi T, Wang D, Ren D, Chen J: Primary Multiple Pulmonary Primitive Neuroectodermal Tumor: Case Report and Literature Review. Medicine 2015, 94(27):e1136.

4. Connolly EM, Gaffney $\varepsilon$, Reynolds JV: Gastrointestinal stromal tumours. The British journal of surgery 2003, 90(10):1178-1186.

5. Willmore-Payne C, Layfield LJ, Holden JA: C-KIT mutation analysis for diagnosis of gastrointestinal stromal tumors in fine needle aspiration specimens. Cancer 2005, 105(3):165-170.

6. Emory TS, Sobin LH, Lukes L, Lee DH, O'Leary TJ: Prognosis of gastrointestinal smooth-muscle (stromal) tumors: dependence on anatomic site. The American journal of surgical pathology 1999, 23(1):82-87.

7. Liu YJ, Yang Z, Hao LS, Xia L, Jia QB, Wu XT: Synchronous incidental gastrointestinal stromal and epithelial malignant tumors. World journal of gastroenterology 2009, 15(16):2027-2031.

8. Maiorana A, Fante R, Maria Cesinaro A, Adriana Fano R: Synchronous occurrence of epithelial and stromal tumors in the stomach: a report of 6 cases. Archives of pathology \& laboratory medicine 2000, 124(5):682-686.

\section{List of abbreviations}

$\begin{array}{ll}\text { AFP } & \text { alpha-fetoprotein } \\ \text { CA 15-3 } & \text { carbohydrate antigen 15-3 } \\ \text { CA 19-9 } & \text { carbohydrate antigen 19-9 } \\ \text { CA 72-4 } & \text { carbohydrate antigen 72-4 } \\ \text { CEA } & \text { carcinoembryonic antigen } \\ \text { CT } & \text { computed tomography } \\ \text { CYFRA 19 } & \text { cytokeratin-19 fragment } \\ \text { GC } & \text { Gastric carcinoma } \\ \text { GIST } & \text { Gastrointestinal stromal tumor } \\ \text { HPF } & \text { high power fields } \\ \text { NGS } & \text { next-generation sequencing } \\ \text { NSE } & \text { neuron-specific enolase } \\ \text { PSA } & \text { prostate-specific antigen } \\ \text { SF } & \text { serum ferritin }\end{array}$

9. Liu SW, Chen GH, Hsieh PP: Collision tumor of the stomach: a case report of mixed gastrointestinal stromal tumor and adenocarcinoma. Journal of clinical gastroenterology 2002, 35(4):332-334

10. Bircan S, Candir O, Aydin S, Baspinar S, Bulbul M, Kapucuoglu N, Karahan $\mathrm{N}$, Ciris $\mathrm{M}$ : Synchronous primary adenocarcinoma and gastrointestinal stromal tumor in the stomach: a report of two cases. The Turkish journal of gastroenterology : the official journal of Turkish Society of Gastroenterology 2004, 15(3):187-191.

11. Lin YL, Tzeng JE, Wei CK, Lin CW: Small gastrointestinal stromal tumor concomitant with early gastric cancer: a case report. World journal of gastroenterology 2006, 12(5):815-817.

12. Wronski M, Ziarkiewicz-Wroblewska B, Gornicka B, Cebulski W, Slodkowski M, Wasiutynski A, Krasnodebski IW: Synchronous occurrence of gastrointestinal stromal tumors and other primary gastrointestinal neoplasms. World journal of gastroenterology 2006, 12(33):5360-5362.

13. Katsoulis IE, Bossi M, Richman PI, Livingstone JI: Collision of adenocarcinoma and gastrointestinal stromal tumour (GIST) in the stomach: report of a case. International seminars in surgical oncology : ISSO 2007, 4:2.

14. Sugimura T, Fujimura S, Baba T: Tumor production in the glandular stomach and alimentary tract of the rat by $\mathrm{N}$-methyl- $\mathrm{N}^{\prime}$-nitro- $\mathrm{N}$-nitrosoguanidine. Cancer research 1970, 30(2):455-465.

15. Cohen A, Geller SA, Horowitz I, Toth LS, Werther JL: Experimental models for gastric leiomyosarcoma. The effects of $\mathrm{N}$-methyl-N'-nitro$\mathrm{N}$-nitrosoguanidine in combination with stress, aspirin, or sodium taurocholate. Cancer 1984, 53(5):1088-1092.

16. Andea AA, Lucas C, Cheng JD, Adsay NV: Synchronous occurrence of epithelial and stromal tumors in the stomach. Archives of pathology \& laboratory medicine 2001, 125(3):318-319.

17. Joensuu $H$, Eriksson M, Sundby Hall K, Hartmann JT, Pink D, Schutte J, Ramadori G, Hohenberger P, Duyster J, Al-Batran SE et al: One vs three years of adjuvant imatinib for operable gastrointestinal stromal tumor: a randomized trial. Jama 2012, 307(12):1265-1272.

18. Fletcher JA, Fletcher CD, Rubin BP, Ashman LK, Corless CL, Heinrich MC: KIT gene mutations in gastrointestinal stromal tumors: more complex than previously recognized? The American journal of pathology 2002, 161(2): 737-738; author reply 738-739.

19. Li CQ, Li RT, Zhang W. Progress in non-invasive detection of liver fibrosis. Cancer Biology Medicine 2018; 15(2): 124-136.

20. Lee FY, Jan YJ, Wang J, Yu CC, Wu CC: Synchronous gastric gastrointestinal stromal tumor and signet-ring cell adenocarcinoma: a case report. International journal of surgical pathology 2007, 15(4):397-400. 